\title{
Nucleon electromagnetic form factors with Wilson fermions
}

QCDSF/UKQCD Collaboration: M. Göckeler ${ }^{a}$, Ph. Hägler ${ }^{b}$, R. Horsley ${ }^{c}$, Y. Nakamura ${ }^{d}$, M. Ohtani ${ }^{a}$, D. Pleiter ${ }^{d}$, P. E. L. Rakow ${ }^{e}$, A. Schäfer ${ }^{a}$, G. Schierholz ${ }^{d f}$, W. Schroers ${ }^{* d}$, H. Stüben ${ }^{g}$, and J. M. Zanotti ${ }^{c}$

${ }^{a}$ Institut für Theoretische Physik, Universität Regensburg, 93040 Regensburg, Germany

${ }^{b}$ Institut für Theoretische Physik T39, Physik-Department der TU München, James-Franck-Strasse, 85747 Garching, Germany

${ }^{c}$ School of Physics, University of Edinburgh, Edinburgh EH9 3JZ, UK

${ }^{d}$ John von Neumann-Institut für Computing NIC / DESY, 15738 Zeuthen, Germany

${ }^{e}$ Theoretical Physics Division, Department of Mathematical Sciences, University of Liverpool, Liverpool L69 3BX, UK

${ }^{f}$ Deutsches Elektronen-Synchrotron DESY, 22603 Hamburg, Germany

${ }^{g}$ Konrad-Zuse-Zentrum für Informationstechnik Berlin, 14195 Berlin, Germany

The nucleon electromagnetic form factors continue to be of major interest for experimentalists and phenomenologists alike. They provide important insights into the structure of nuclear matter. For a range of interesting momenta they can be calculated on the lattice. The limiting factor continues to be the value of the pion mass. We present the latest results of the QCDSF collaboration using gauge configurations with two dynamical, non-perturbatively improved Wilson fermions at pion masses as low as $350 \mathrm{MeV}$.

DESY 07-153

Edinburgh 2007/23

The XXV International Symposium on Lattice Field Theory

July 30 - August 42007

Regensburg, Germany

${ }^{*}$ Speaker. 


\section{Introduction}

Protons and neutrons constitute most of the material world around us. This makes them the most important particles subject to the strong interaction. Their electromagnetic form factors were among the first quantities investigated in hadron structure and they are known for several decades. For a recent review on the status of experiment and phenomenology consult [1]. Nonetheless, despite all the scrutiny they are still subject to surprises. Recently, a series of experiments has been performed at Jefferson Lab [2]. These have revealed surprising new features of the form factors namely, that their ratio $F_{2}\left(Q^{2}\right) / F_{1}\left(Q^{2}\right)$ does not behave as was expected from previous experiments and as predicted by perturbative QCD. Resolving this mystery requires model-independent nonperturbative methods. Lattice QCD provides such a method without model assumptions.

A theoretical explanation of the behavior of the form factor ratio has been suggested in [3]. In fact, lattice calculations have recently been found to show similar behavior $[4,5,6]$. This is an example of how lattice calculations can give important feedback to phenomenology and experiment already today.

This paper focusses on the calculation of the behavior of the electromagnetic vector and axial form factors of the isovector combination $p-n$, i.e., the difference between up- and down-quarks unless explicitely stated otherwise. Due to isospin symmetry the disconnected contributions cancel in this situation and there is no additional systematic error other than the usual lattice systematics. From the Lorentz-invariant expansions of the vector and the axial currents, we define the form factors via

$$
\begin{aligned}
& \left\langle N\left(\vec{p}^{\prime}\right)\left|J^{\mu}\right| N(\vec{p})\right\rangle=\bar{u}\left(\vec{p}^{\prime}\right)\left(\gamma^{\mu} F_{1}\left(Q^{2}\right)+\frac{\mathrm{i} q_{\alpha}}{2 m_{N}} \sigma^{\alpha \mu} F_{2}\left(Q^{2}\right)\right) u(\vec{p}), \\
& \left\langle N\left(\vec{p}^{\prime}\right)\left|A^{\mu}\right| N(\vec{p})\right\rangle=\bar{u}\left(\vec{p}^{\prime}\right)\left(\gamma^{\mu} G_{A}\left(Q^{2}\right)+\frac{q^{\mu}}{2 m_{N}} G_{P}\left(Q^{2}\right)\right) \gamma_{5} u(\vec{p}),
\end{aligned}
$$

with $\vec{p}^{\prime}(\vec{p})$ being the initial (final) nucleon momentum, $q=p^{\prime}-p, Q^{2}=-q^{2}$ the virtual momentum transfer, and $m_{N}$ being the nucleon mass.

We employ two flavors of dynamical Wilson-clover fermions with pion masses as low as $m_{\pi}=$ $350 \mathrm{MeV}$ [7]. The lattice spacing varies between $a=0.065 \ldots 0.08 \mathrm{fm}$ where the scale has been set by $r_{0}=0.45 \mathrm{fm}$. This technology has the advantage that it extends the successful calculations of the past decade without conceptual problems like square roots of sea quarks, flavor and/or taste breaking, residual masses or absence of unitarity away from the continuum limit. The technology to extract form factors has been covered in detail in $[8,9]$.

\section{Vector form factors}

To investigate the large-distance behavior of the vector form factors we adopt the following parameterizations of $F\left(Q^{2}\right)$ as functions of $Q^{2}$ :

$$
\begin{aligned}
& F_{1}\left(Q^{2}\right)=\frac{A}{1+c_{12} Q^{2}+c_{14} Q^{4}}, \\
& F_{2}\left(Q^{2}\right)=\frac{1+\kappa}{1+c_{22} Q^{2}+c_{26} Q^{6}} .
\end{aligned}
$$


These parameterizations have been inspired by $[3,10]$ by neglecting logarithmic corrections. Such perturbative logarithms are not expected to play a role for the energy range $Q^{2}<4 \mathrm{GeV}^{2}$ we are investigating. The normalization factor, $A$, is used to fix the renormalization constant. Due to charge conservation, we must have $F_{1}\left(Q^{2}\right) \equiv 1$ which fixes the operator renormalization. The parameterizations Eqs. (2.1) have a couple of important properties:

- The fulfill the superconvergence relation

$$
\int_{-4 m_{\pi}^{2}}^{-\infty} d Q^{2} \operatorname{Im} F_{1,2}\left(Q^{2}\right)=0, \quad \int_{-4 m_{\pi}^{2}}^{-\infty} d Q^{2} Q^{2} \operatorname{Im} F_{2}\left(Q^{2}\right)=0 .
$$

- They fulfill the expected asymptotic behavior

$$
F_{1} \propto 1 /\left(Q^{2}\right)^{2}, \quad F_{2} \propto 1 /\left(Q^{2}\right)^{3} .
$$

- They should exhibit an effective resonance pole for negative $Q^{2}$. In particular, we will verify below that the location of the pole lies at the vector meson mass. This serves as a consistency check between our lattice calculation and phenomenology.

Alternatively, one can use dipole and tripole-type fit formulae $[5,6]$. We believe, however, that the parameterizations in Eqs. (2.1) describe the physics better. It is important to point out that the lattice data does not cover a sufficiently large range of $Q^{2}$ values to allow us to favor one form over another. Hence, we require additional phenomenological input and perform consistency checks by probing the location of the poles of $F_{1}\left(Q^{2}\right)$ in Eq. (2.1).

From the parameterizations in Eq. (2.1) we can obtain the charge radii via a Taylor expansion

$$
F_{i}\left(Q^{2}\right)=F_{i}(0)\left(1-\frac{1}{6}\left\langle r_{i}^{2}\right\rangle Q^{2}+\mathscr{O}\left(Q^{4}\right)\right), \quad\left\langle r_{i}^{2}\right\rangle=6 c_{i 2} .
$$

It is evident that the radius only depends on $c_{12} / c_{22}$, but not on the higher coefficients of the powers $Q^{4} / Q^{6}$.

The first question we address is the flavor-dependence of the connected contribution to the charge radius, $\left\langle r_{1}^{2}\right\rangle$. Figure 1 shows the different charge radii as a function of the pion mass at a fixed value of $\beta=5.29$. For large pion masses the difference is below the statistical uncertainty. As the pion mass decreases, however, the difference starts to become significant. This is an excellent example of why going towards sufficiently light quarks is crucial to study phenomena related to hadron structure.

As it is evident from Eq. (2.1) the expression for $F_{1}\left(Q^{2}\right)$ has in general two poles in the complex $Q^{2}$ plane. Both poles can either be complex — with a common real part — or real — with a lower and an upper mass. From the vector meson dominance model we expect the lower real pole and the real part of the complex poles to be around the vector meson mass. Figure 2 shows the location of the lower real/real part of the poles divided by the vector meson mass at each working point. For u-quarks we find mostly two real poles with a few complex poles at some working points. For d-quarks we find complex poles exclusively. While the real poles are indeed compatible with the vector meson mass, the real parts of the complex poles scatter more strongly and tend to 


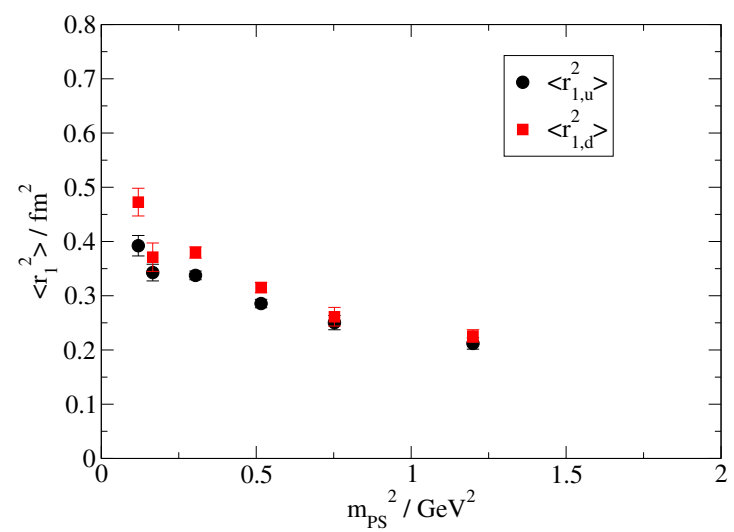

Figure 1: Difference in connected part of the charge radii for up- and down-quarks vs. the pion mass.
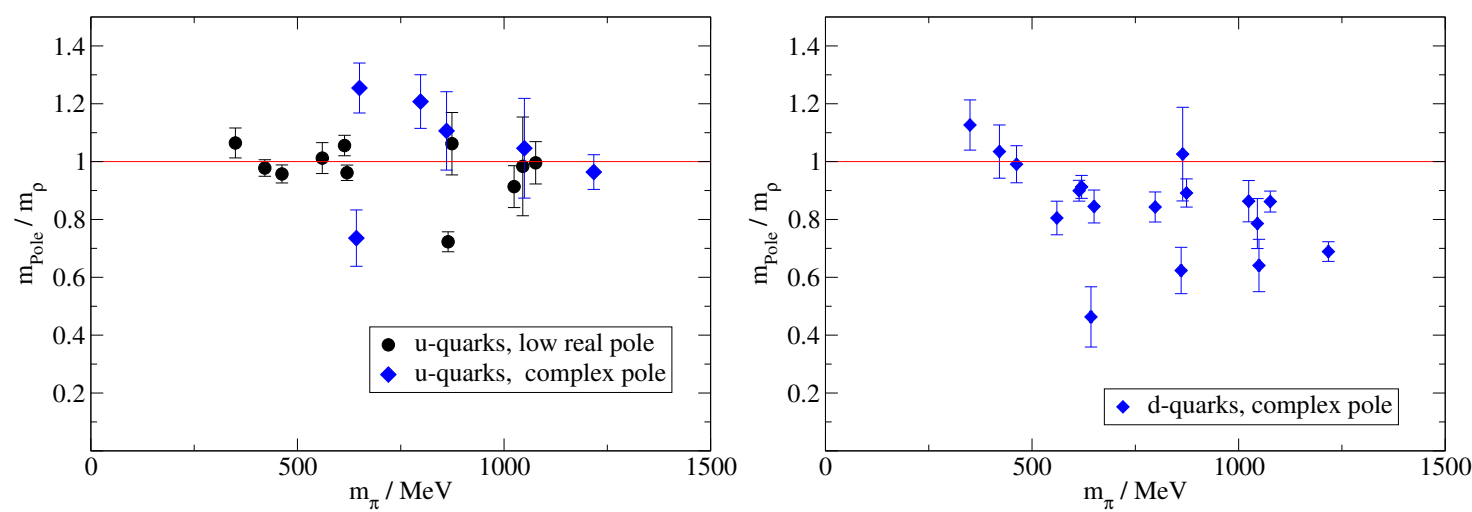

Figure 2: Location of the real part of the pole masses for u/d quarks (left/right panel). For u-quarks we find mostly two real poles and complex poles at a few working points. For d-quarks we find only complex poles.

lie somewhat lower. Understanding this phenomenon better would further improve our picture of the nucleon.

The dependence of the charge radii on the pion mass has been studied by different groups [11, 8]. In the following we focus on the small-scale expansion (SSE) given in [8]. Figure 3 shows the lattice results for $\left\langle r_{1}^{2}\right\rangle$ together with the experimental point denoted by a star. The dashed curve is the SSE expression with phenomenologically reasonable values for the parameters. The curve grows to infinity as the pion mass goes to zero. Furthermore, it vanishes at a finite value of the pion mass. The latter behavior is unphysical, so we conclude that (not surprisingly) the SSE expression at the order considered does not describe this quantity over the entire range of pion masses available.

Figure 4 shows the results of a combined fit of all lattice data for $\left\langle r_{2}^{2}\right\rangle$ and $\kappa^{v}$. In these expressions there are four parameters that we fit. The error bands show the statistical errors and the systematic error due to higher orders in the chiral expansion as determined by varying the individual fit parameters and checking the stability of the result [12]. For $\left\langle r_{2}^{2}\right\rangle$ the extrapolation misses the experimental data point, while for $\kappa^{v}$ we find that the extrapolation is compatible with the experimental data point. It is premature to conclude that the expansion fails for $\left\langle r_{2}^{2}\right\rangle$ as it did for $\left\langle r_{1}^{2}\right\rangle$ since the uncertainties of the input parameters may be underestimated. Nonetheless, the range of appli- 


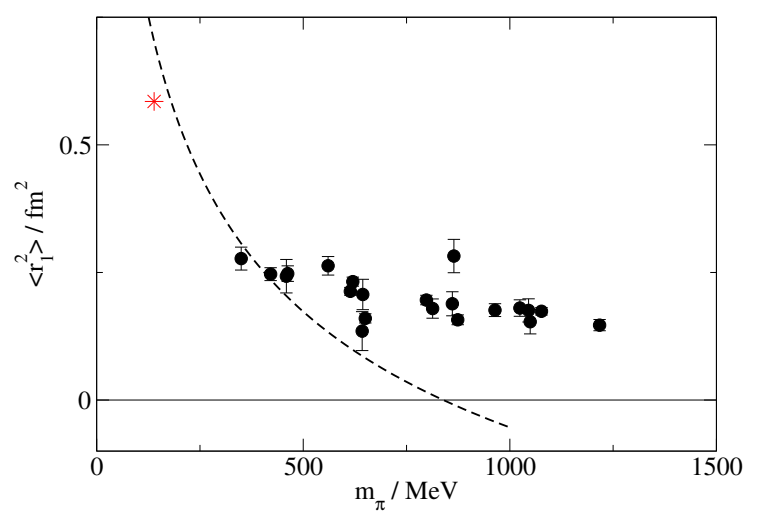

Figure 3: Isovector radius $\left\langle r_{1}^{2}\right\rangle$ as a function of the pion mass. The experimental value is denoted by a star, the dashed line is the expression obtained from the SSE.
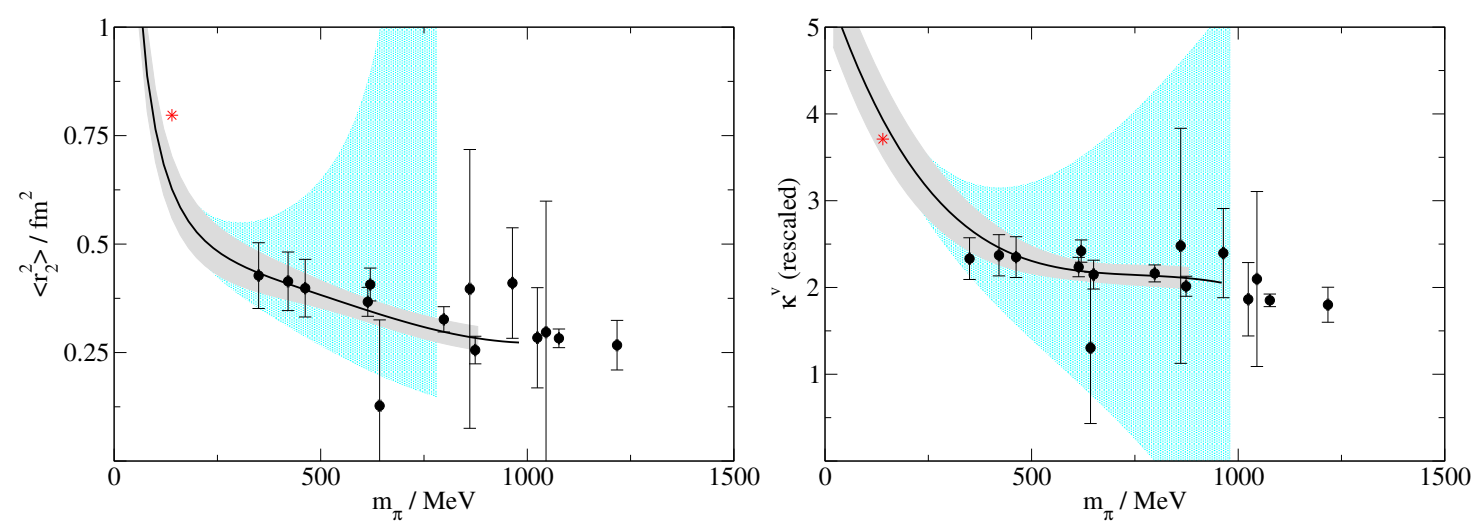

Figure 4: Isovector radius $\left\langle r_{2}^{2}\right\rangle$ (left panel) and anomalous magnetic moment $\kappa^{v}$ (right panel) from a combined fit of all lattice data. The rescaling has been explained in Ref. [8]. The experimental values are denoted by stars. The error bands are statistical (shaded gray) and systematical (dotted cyan).

cability of the chiral expansion is limited and further study is needed to accumulate sufficiently accurate data points at sufficiently small pion masses.

\section{Axial form factors}

Analogous to the form factors $F_{1}\left(Q^{2}\right)$ and $F_{2}\left(Q^{2}\right)$ the axial form factor, $G_{A}\left(Q^{2}\right)$, and the induced pseudoscalar form factor, $G_{P}\left(Q^{2}\right)$, can be calculated. For a review on experimental methods and phenomenological parameterizations see [13]. The axial form factor is usually fitted using a dipole ansatz

$$
G_{A}\left(Q^{2}\right)=\frac{g_{A}}{\left(1+Q^{2} / M_{A}^{2}\right)^{2}},
$$

with the axial coupling $g_{A}$ being one of the milestones of lattice QCD calculations [14], see also [15]. The induced pseudoscalar coupling $G_{P}\left(Q^{2}\right)$ is well understood and can be fitted using a pion-pole ansatz for sufficiently small $m_{\pi}$ and $Q^{2}$, see [13]:

$$
G_{P}\left(Q^{2}\right)=\frac{4 m_{N} g_{\pi N} f_{\pi}}{m_{\pi}^{2}+Q^{2}}-\frac{2}{3} g_{A} m_{N}^{2}\left\langle r_{A}^{2}\right\rangle+\mathscr{O}\left(Q^{2}, m_{\pi}^{2}\right) .
$$



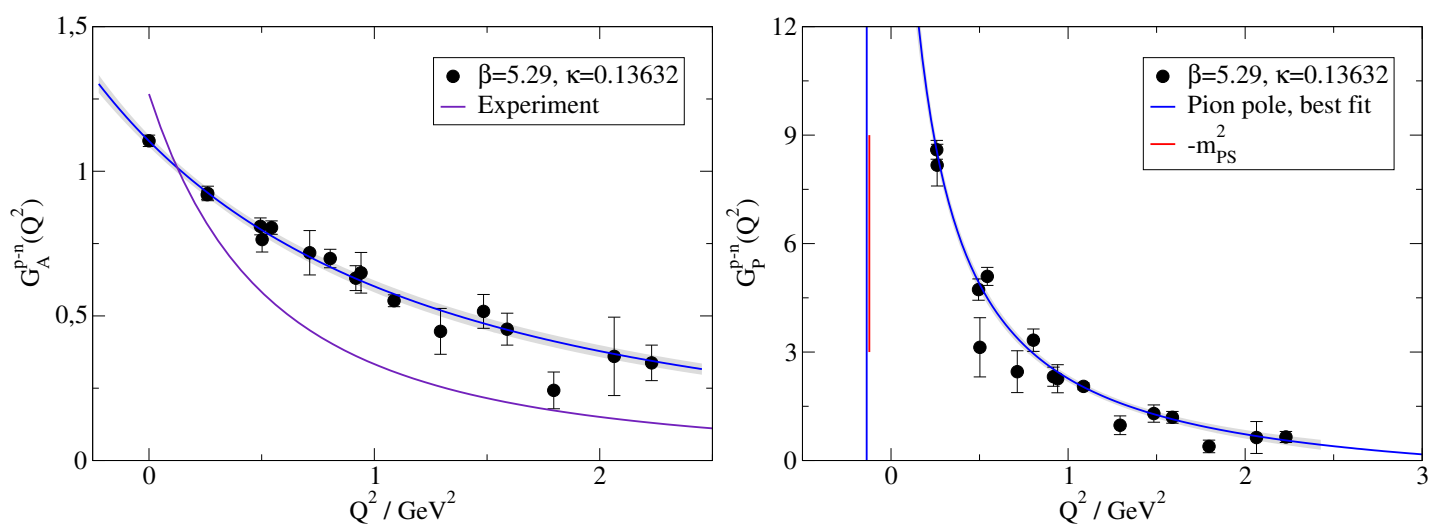

Figure 5: Isovector axial form factors for a sample working point at our lowest pion mass of $m_{\pi}=350 \mathrm{MeV}$. The axial form factor (left panel) is fitted using a dipole form, the experimental best dipole fit is included. The pseudoscalar form factor (right panel) is fitted using a pion pole expression with the evaluated pseudoscalar mass being shown for comparison.

We want to test this ansatz by verifying that the position of the pole is exactly at the position of the pion mass at this working point.

Our results for $G_{A}\left(Q^{2}\right)$ and $G_{P}\left(Q^{2}\right)$ are shown in Fig. 5 for a sample working point at our lowest pion mass of $m_{\pi}=350 \mathrm{MeV}$. The axial coupling fitted with a dipole is compared to the corresponding experimental result. It is evident that the lattice curve is flatter, implying that the heavy quarks on our lattice build a smaller nucleon than Nature - a phenomenon that has been observed previously for the vector form factors, cf. Figs. 3 and 4. The induced pseudoscalar form factor indeed exhibits a pole at the location of the measured pion mass - as indicated in the plot by a vertical bar. It is evident that the pole obtained from the fitted pion-pole parameterization is fully consistent with the value of the pion mass. Hence, our fit seems to be remarkably consistent with the expression Eq. (3.2).

\section{Summary}

We have successfully measured the vector and the axial vector form factors. For the vector form factors a variety of chiral expansions is available. The radius $\left\langle r_{1}^{2}\right\rangle$ cannot be described well, while the situation for $\left\langle r_{2}^{2}\right\rangle$ and $\kappa^{v}$ is more favorable. The axial form factor $G_{A}\left(Q^{2}\right)$ turns out to be described well by a dipole formula — just like the experimental data - but the curve is flatter indicating that also in this situation a proper chiral extrapolation is essential. We verified that the induced pseudoscalar form factor, $G_{P}\left(Q^{2}\right)$, is described excellently by the pion pole picture already at $m_{\pi}=350 \mathrm{MeV}$.

We conclude by pointing out that dynamical clover fermions provide a viable technology to treat QCD with light quarks. The currently running simulations may extend down to pion masses as low as $m_{\pi}=250 \mathrm{MeV}$ within the next year. We are optimistic to reach $m_{\pi}=200 \mathrm{MeV}$ by the end of this decade. 


\section{Acknowledgments}

The numerical calculations have been performed on the Hitachi SR8000 at LRZ (Munich), the BlueGene/L and the Cray T3E at EPCC (Edinburgh) [16], the BlueGene/Ls at NIC/JFZ (Jülich) and KEK (by the Kanazawa group as part of the DIK research program) and on the APEmille and apeNEXT at NIC/DESY (Zeuthen). This work was supported in part by the DFG under contract FOR 465 (Forschergruppe Gitter-Hadronen-Phänomenologie and Emmy-Noether program) and by the EU Integrated Infrastructure Initiative Hadron Physics (I3HP) under contract number RII3-CT2004-506078. W.S. thanks Wolfgang Bietenholz for valuable discussions.

\section{References}

[1] V. Punjabi et al., Phys. Rev. C 71 (2005) 055202 [Erratum-ibid. C 71 (2005) 069902]; J. Arrington, C. D. Roberts and J. M. Zanotti, J. Phys. G 34 (2007) S23.

[2] M. K. Jones et al. [Jefferson Lab Hall A Collaboration], Phys. Rev. Lett. 84 (2000) 1398; O. Gayou et al. [Jefferson Lab Hall A Collaboration], Phys. Rev. Lett. 88 (2002) 092301; O. Gayou et al., Phys. Rev. C 64 (2001) 038202.

[3] A. V. Belitsky, X. D. Ji and F. Yuan, Phys. Rev. Lett. 91 (2003) 092003.

[4] J. W. Negele et al. [LHPC Collaboration], Nucl. Phys. Proc. Suppl. 129 (2004) 910; M. Göckeler et al. [QCDSF Collaboration], Nucl. Phys. Proc. Suppl. 135 (2004) 156; M. Göckeler et al., Eur. Phys. J. A 32 (2007) 445.

[5] M. Göckeler et al., PoS LAT2006 (2006) 120.

[6] W. Schroers et al., arXiv:0709.3370.

[7] M. Göckeler et al., PoS LAT2006 (2006) 179.

[8] M. Göckeler, T. R. Hemmert, R. Horsley, D. Pleiter, P. E. L. Rakow, A. Schäfer and G. Schierholz [QCDSF Collaboration], Phys. Rev. D 71 (2005) 034508.

[9] M. Göckeler, R. Horsley, D. Pleiter, P. E. L. Rakow, A. Schäfer, G. Schierholz and W. Schroers [QCDSF Collaboration], Phys. Rev. Lett. 92 (2004) 042002; Ph. Hägler, J. Negele, D. B. Renner, W. Schroers, Th. Lippert and K. Schilling [LHPC collaboration], Phys. Rev. D 68 (2003) 034505.

[10] M. A. Belushkin, H. W. Hammer and U. G. Meissner, Phys. Rev. C 75 (2007) 035202.

[11] S. I. Ando, J. W. Chen and C. W. Kao, Phys. Rev. D 74 (2006) 094013; M. Diehl, A. Manashov and A. Schäfer, Eur. Phys. J. A 31 (2007) 335; B. Kubis and U. G. Meissner, Nucl. Phys. A 679 (2001) 698; P. Wang, D. B. Leinweber, A. W. Thomas and R. D. Young, Phys. Rev. D 75 (2007) 073012; D. B. Leinweber, A. W. Thomas and R. D. Young, Phys. Rev. Lett. 86 (2001) 5011.

[12] U. Meissner, private communication.

[13] V. Bernard, L. Elouadrhiri and U. G. Meissner, J. Phys. G 28 (2002) R1.

[14] R. G. Edwards et al. [LHPC Collaboration], Phys. Rev. Lett. 96 (2006) 052001; A. Ali Khan et al., Phys. Rev. D 74 (2006) 094508.

[15] W. Schroers, Eur. Phys. J. A 31 (2007) 784.

[16] UKQCD Collaboration, C. R. Allton et al., Phys. Rev. D 65 (2002) 054502. 